\title{
Bladder Rupture following Conversion to Enteric Drainage after Pancreatic Transplantation
}

\author{
Vikas Srivastava $^{a} \quad$ George Passaris $^{a}$ Rajiv Juneja ${ }^{a}$ \\ Mark Siddins $^{b}$ Jeffrey A.J. Barbara ${ }^{a}$ \\ ${ }^{a}$ Renal Unit, and ${ }^{b}$ Department of Urology, Flinders Medical Centre and \\ Flinders University, Adelaide, S.A., Australia
}

\section{Key Words}

Pancreatic transplantation · Bladder rupture

\begin{abstract}
Complications associated with bladder-drained pancreatic transplant are not uncommon and include urinary tract infections and reflux pancreatitis. Bladder rupture with peritoneal leak is a rare complication after pancreatic transplantation and can present as an acute abdomen with rapidly deteriorating renal function. We describe the first case of a urine leak into the peritoneal cavity occurring after conversion from bladder to enteric drainage. A high index of suspicion is required to diagnose such a complication.
\end{abstract}

\section{Background}

Bladder-drained pancreatic recipients experience a relatively high incidence of metabolic and urologic complications resulting in conversion to enteric drainage in about 15-30\% of cases. Spontaneous rupture of the bladder after enteric conversion of a bladder-drained pancreatic transplant has not been previously reported.

\section{Case Report}

A 44-year-old man with diabetes mellitus type 1 and end-stage renal failure receiving haemodialysis for four years received a sequential pancreatic transplant after renal transplant in March 2002 and July 2001, respectively. Immunosuppression after transplant included tacrolimus, mycophenolate and prednisolone. The exocrine secretions of the transplanted pancreas drained into the urinary bladder. Due to recurrent urinary tract infection and significant dysuria despite urinary alkalinisation, he underwent pancreatic duodenojejunostomy in June 2003. The following three years were complication free. 
In April 2006, he presented to the Emergency Department with generalised abdominal pain associated with non-bilious vomits. He was febrile at $38.6^{\circ} \mathrm{C}$, hypotensive and tachycardic with signs of peritonism. The creatinine had increased to $286 \mu \mathrm{mol} / \mathrm{l}$ from $170 \mu \mathrm{mol} / \mathrm{l}$ and the white cell count was $20 \times 10^{9} /$ l. Plain X-ray and CT of the abdomen were non-diagnostic. Empirical therapy with intravenous antibiotics and fluids was administered after an indwelling urinary catheter had been inserted at presentation. He improved rapidly and renal function returned to baseline within $24 \mathrm{~h}$ of admission. A very similar presentation occurred two months later. Clinical and biochemical improvement once again occurred quickly and an outpatient colonoscopy and barium swallow returned normal findings.

In December 2006, he presented for a third occasion with abdominal pain and vomiting to the renal outpatients department. The previous evening, he had been lifting a sack of potatoes and experienced some discomfort in his perineum. On examination there were features of peritonism and ascites was detected. The creatinine had risen to $348 \mu \mathrm{mol} / \mathrm{l}$ from his usual baseline and on the following day it was $490 \mu \mathrm{mol} / \mathrm{l}$. The non-contrast CT of the abdomen confirmed gross ascites. A diagnostic abdominal paracentesis was performed and the creatinine in the ascitic fluid proved to be very similar to serum $(501 \mu \mathrm{mol} / \mathrm{l}$ compared to $490 \mu \mathrm{mol} / \mathrm{l})$. The urinary creatinine at this time was $6.24 \mathrm{mmol} / \mathrm{l}$. A urinary catheter was inserted into the bladder with a rapid improvement in the serum creatinine and a reduction in the volume of ascites. A provisional diagnosis of urinary bladder leak

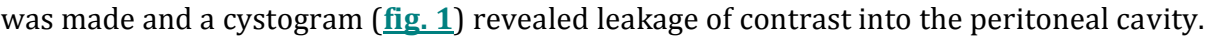

In the operating theatre, the leak was confirmed with repeat cystography. An omental patch was mobilised from the transverse mesocolon and placed over the dome of the bladder (fig. 2). A bladder biopsy showed focal scarring with no dysplasia or malignancy. A cystogram repeated two months later did not reveal any extravasation of contrast into the peritoneal cavity. There have been no complications relating to this procedure. In April 2009, he presented with hyperglycaemia and has become dependent on exogenous insulin.

\section{Discussion}

Bladder-drained pancreatic transplants have been associated with complications such as urinary infection and dysuria which can lead to a requirement for enteric drainage [1]. However, bladder rupture after conversion from bladder to enteric drainage has so far not been reported in the literature. This occurred in our case three years after enteric conversion, and the diagnosis was not established until the third acute presentation when a urinary catheter was not initially placed. In our opinion, early urinary catheterisation with the first two presentations allowed the bladder to rest at low volume and resulted in a temporary repair. With the final presentation, the urinary effluent escaped from the bladder directly into the peritoneal cavity resulting in a 'reverse peritoneal dialysis' with recycling or reabsorption of creatinine and a rapid increase in serum creatinine. Metabolic acidosis and hyperkalaemia were also developing quickly. The placement of an indwelling urinary catheter halted this recycling phenomenon.

Urinary bladder rupture is a rare condition but one in which a pathological basis can be identified in most cases [2,3]. Tarrass et al. [4] identified 23 cases of renal failure following bladder rupture and these were mainly due to intrapelvic gynaecological operations, eosinophilic cystitis, blunt trauma to the abdomen, anatomical outflow obstruction and in association with indwelling catheters [5-10]. The clinical presentation can be variable but often includes oliguria, acute kidney injury and ascites with or without peritonitis. Early diagnosis is important as the condition can be associated with high mortality. 
Whilst routine imaging techniques allow a diagnosis of bladder rupture to be made in the majority of cases, its rare nature demands a high level of clinical suspicion where this constellation of clinical features exists [11]. For a definitive diagnosis, conventional cystography is the gold standard [12] (95\% sensitivity) although CT cystograms are considered of equal value [13]. Intravenous urography, duplex sonography, cystoscopy and laparoscopy have also led to the diagnosis in some cases. The management of bladder rupture depends on the site of the rupture. For extraperitoneal rupture, bladder catheterisation alone has been considered safe and effective [14], but for intraperitoneal bladder rupture, open repair with an omental patch is favoured and this is especially the case with recurrent bladder rupture.

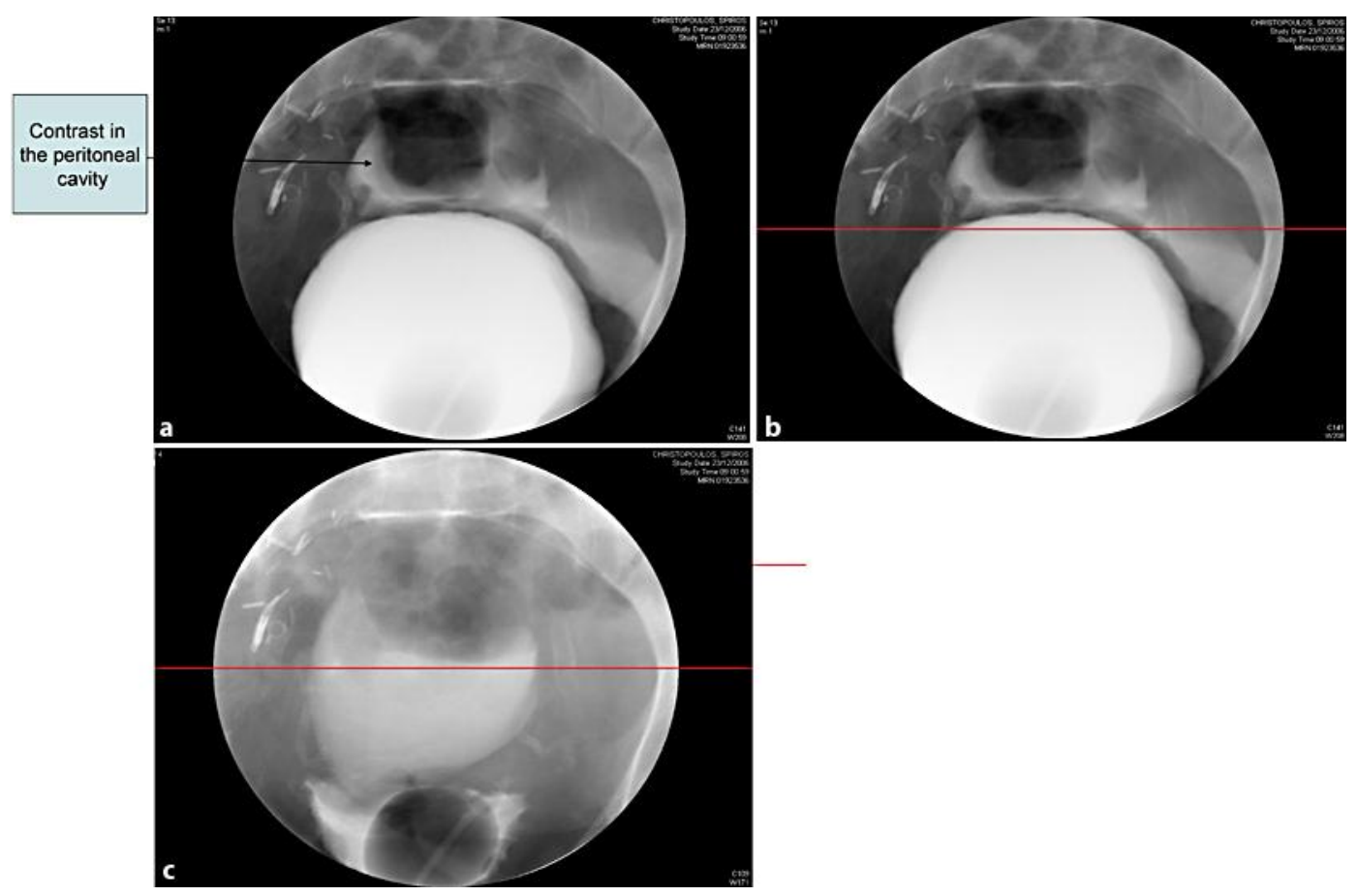

Fig. 1. Cystogram with contrast in the urinary bladder and early escape of contrast superior to the bladder. 

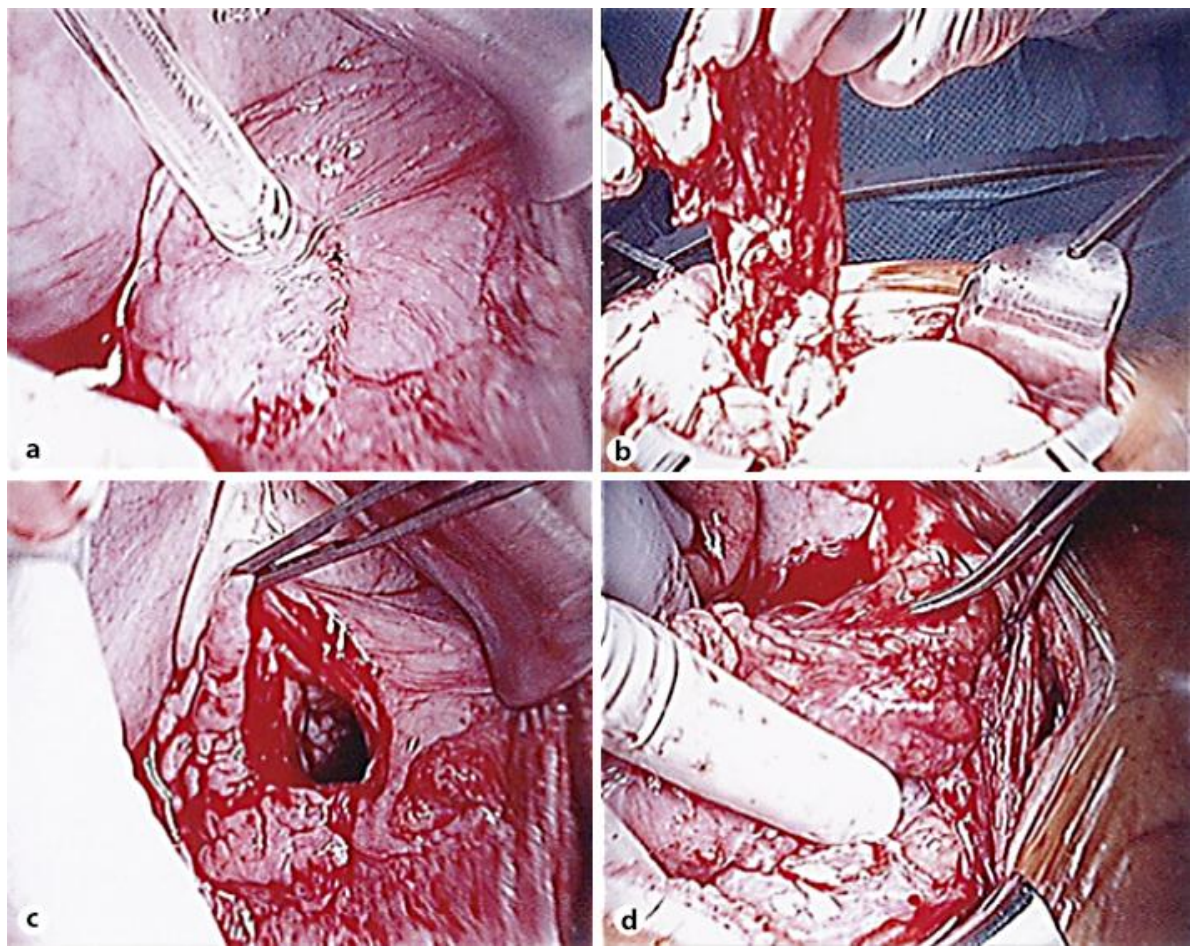

Fig. 2. a Urinary bladder located. b Omentum mobilised. c Rupture on the dome identified. d Omental patch sited.

\section{References}

1 Troppmann C: Complications after pancreas transplantation. Curr Opin Transplant 2010;15:112-118.

-2 Bastable JR, De Jode LR, Warren RP: Spontaneous rupture of the bladder. Br J Urol 1959;31:78-86.

-3 Desmond AD, Woolfenden KA, Evans CM: The importance of urodynamic investigations following spontaneous rupture of the bladder. J Urol 1983;129:140-142.

4 Tarrass F, Sirajedine K, Koenig J, Leroy F, Nys S, Colomb H: Acute renal failure following ruptured bladder after electroresection of a polyp. Hong Kong J Nephrol 2006;8:36-39.

-5 Fujikawa K, Miyamoto T, Ihara Y, Matsui Y, Takeuchi H: High incidence of severe urologic complications following radiotherapy for cervical cancer in Japanese women. Gynecol Oncol 2001;80:21-23.

-6 Addar MH, Stuart GCE, Nation JG, Shumsky AG: Spontaneous rupture of the urinary bladder: a late complication of radiotherapy - case report and review of the literature. Gynecol Oncol 1996;62:314316.

7 Nishimura T, Suzuki K, Iijima M, et al: Spontaneous rupture of bladder diverticulum after postoperative radiotherapy for carcinoma of the uterine cervix: a case report. Radiat Med 2000;18:261-265.

8 Lynn SJ, Mark SD, Searle M: Idiopathic spontaneous bladder rupture in an intoxicated patient. Clinical Nephrol 2003;6:430-432.

-9 Basavaraj DR, Zachariah KK, Feggetter JGW: Acute abdomen - remember spontaneous perforation of the urinary bladder. J R Coll Surg Edin 2001;46:316-317.

10 Chow KM, Lam CM, Szeto CC: Pseudo-renal failure following total abdominal hysterectomy. J Nephrol 2005;18:442-446.

11 Carmon M, Nissan A, Pappo I, Perlberg S, Sector D, Haskel Y: Spontaneous rupture of the urinary bladder complicated by extensive fasciitis. The importance of a high index of suspicion. Urol Int 1994;52:38-40.

12 Caroll PR, Aninch JW: Major bladder trauma: the accuracy of cystography. J Urol 1983;130:887-888. 
13 Sandler CM, Hall JT, Rodriguez MB, Corriere JN: Bladder injury in blunt pelvic trauma. Radiology 1986;158:633-638.

14 Corriere JN, Sandler CM: Management of the ruptured bladder: seven years of experience with 111 cases. J Trauma 1986;26:830-833. 